\title{
On the Existence Conditions of Surface Spin Wave Modes in (Ga,Mn)As Thin Films
}

\author{
H. PUSZKARSKI ${ }^{a, *}$ AND P. TOMCZAK ${ }^{b}$ \\ ${ }^{a}$ Surface Physics Division, Faculty of Physics, Adam Mickiewicz University, Umultowska 85, 61-614 Poznań, Poland \\ ${ }^{b}$ Quantum Physics Division, Faculty of Physics, Adam Mickiewicz University, \\ Umultowska 85, 61-614 Poznań, Poland \\ (Received December 18, 2015)
}

\begin{abstract}
Spin-wave resonance is a newly emerged method for studying surface magnetic anisotropy and surface spinwave modes in $(\mathrm{Ga}, \mathrm{Mn}) \mathrm{As}$ thin films. The existence of surface spin-wave modes in (Ga,Mn)As thin films has recently been reported in the literature; surface spin-wave modes have been observed in the in-plane configuration (with variable azimuth angle $\varphi_{M}$ between the in-plane magnetization of the film and the surface [100] crystal axis), in the azimuth angle range between two in-plane critical angles $\varphi_{\mathrm{c} 1}$ and $\varphi_{\mathrm{c} 2}$. We show here that cubic surface anisotropy is an essential factor determining the existence conditions of the above-mentioned surface spin-wave modes: conditions favorable for the occurrence of surface spin-wave modes in a (Ga,Mn)As thin film in the inplane configuration are fulfilled for those azimuth orientations of the magnetization of the sample that lie around the hard axes of cubic magnetic anisotropy. This implies that a hard cubic anisotropy axis can be regarded in (Ga,Mn)As thin films as an easy axis for surface spin pinning.
\end{abstract}

DOI: 10.12693/APhysPolA.129.117

PACS/topics: 75.50.Pp, 76.50.+g, 75.70.-i, 75.30.Ds

\section{Introduction}

Recent studies of the electronic structure of (Ga,Mn)As by spin-resolved photoemission [1] indicate that the surface region of a $(\mathrm{Ga}, \mathrm{Mn})$ As sample can be regarded as a layer with properties qualitatively different from those of the underlying bulk. In particular, the authors of the cited paper demonstrate that the surface of this material has a ferromagnetic character even at room temperature; consequently, in these temperature conditions a $(\mathrm{Ga}, \mathrm{Mn})$ As sample will include a ferromagnetic phase confined to a very thin surface region. The above-mentioned finding can be a precious contribution to the ongoing debate $[2,3]$ on the essential mechanisms increasing the Curie temperature in $(\mathrm{Ga}, \mathrm{Mn}) \mathrm{As}$, since in the light of the cited paper [1] in-depth studies of the nature of the surface ferromagnetism in this material could bring us closer to the understanding of the processes that might increase its Curie temperature even to the room temperature range.

The present study is devoted to spin-wave resonance (SWR), a very effective experimental method for the study of ferromagnetic properties of $(\mathrm{Ga}, \mathrm{Mn}) \mathrm{As}$ thin films, and their surface in particular. It allows full characterization of the surface magnetic anisotropy, which, as we demonstrate here, provides the basis for the determination of the existence conditions of magnetic (spinwave) modes localized at the surface of a $(\mathrm{Ga}, \mathrm{Mn}) \mathrm{As}$ sample. We believe that this result is a step forward in exploring the differences in ferromagnetic properties between the surface and bulk of this material.

*corresponding author; e-mail: henpusz@amu.edu.pl

\section{SWR surface inhomogeneity model}

The existence of surface spin-wave modes (SSWMs) in (Ga,Mn)As thin films was first reported by Liu et al. [4], which observed SSWMs in both the out-of-plane configuration, with variable polar angle $\vartheta_{M}$ between the magnetization of the film and its surface normal, and the inplane configuration, with variable azimuth angle $\varphi_{M}$ between the in-plane magnetization of the film and the [100] crystal axis. In the former case SSWMs only exist when the polar angle $\vartheta_{M}$ is larger than a certain angle - the out-of-plane critical angle - while in the in-plane configuration SSWMs are observed in the azimuth angle range between two in-plane critical angles.

These experimental findings are interpreted theoretically in our recent papers [5-7], in which we propose an appropriate model of surface anisotropy that both explains the existence of critical angles and allows to determine the conditions of existence of SSWMs in full agreement with the experimental data. In our model of surface anisotropy we use the concept of surface pinning parameter $A_{\text {surf }}$, which describes the freedom of precessing surface spins in relation to the freedom of precessing bulk spins. We have determined the functions $A_{\text {surf }}\left(\vartheta_{H}\right)$ and $A_{\text {surf }}\left(\varphi_{M}\right)$ describing the configuration dependence of the surface parameter in the out-of-plane and in-plane configurations, respectively. The explicit formula for the in-plane surface parameter reads [6]:

$$
\begin{gathered}
A_{\text {surf }}\left(\varphi_{M}\right)=1+a_{\text {iso }}+a_{\text {uni }} \sin ^{2}\left(\varphi_{M}-45^{\circ}\right) \\
\quad+a_{\text {cub }}\left[\left(3+\cos 4 \varphi_{M}\right)+\left(3+\cos 4 \varphi_{M}\right)^{4}\right] .
\end{gathered}
$$

A satisfactory interpretation of the experimental SWR spectra obtained in the in-plane configuration [4] is achieved with the surface parameter described by the series presented above in Eq. (1) (found in an investigation 
discussed in detail in [6]) with the following values of the series coefficients $a_{\text {iso }}, a_{\text {uni }}$ and $a_{\text {cub }}$ :

$$
a_{\text {iso }}=0.1058 ; \quad a_{\text {uni }}=0.027 ; \quad a_{\text {cub }}=-0.0023 .
$$

The series coefficients in Eq. (1) describe the contributions of different surface anisotropy components to the surface spin pinning in $(\mathrm{Ga}, \mathrm{Mn})$ As thin films: $a_{\text {iso }}$ is the isotropic contribution to the pinning, $a_{\text {uni }}$ is related to the uniaxial component, and $a_{\text {cub }}$ describes the pinning due to the cubic anisotropy.

In our model the critical angles are determined by the condition $A_{\text {surf }}\left(\varphi_{M}\right)=1$, and the configuration angles for which SSWMs exist by the condition $A_{\text {surf }}\left(\varphi_{M}\right)>1$. Based on these conditions, in the present paper we will discuss the impact of each of the surface anisotropy components appearing in Eq. (1), and provide a physical interpretation of their respective roles in the generation of SSWMs. To this end we will use a surface pinning diagram built in the polar coordinate system; using this pinning diagram we will obtain a graphical representation of the configuration dependence of the pinning felt by the surface spins.

\section{Surface pinning diagram vs. SWR spectra}

The surface pinning diagram is a planar map showing how the dynamics of surface spins in a $(\mathrm{Ga}, \mathrm{Mn})$ As thin film changes with their orientation with respect to specific crystal axes that characterize the surface structure. The pinning diagram is based on a polar coordinate system lying in the plane of the film surface (see Fig. 1).

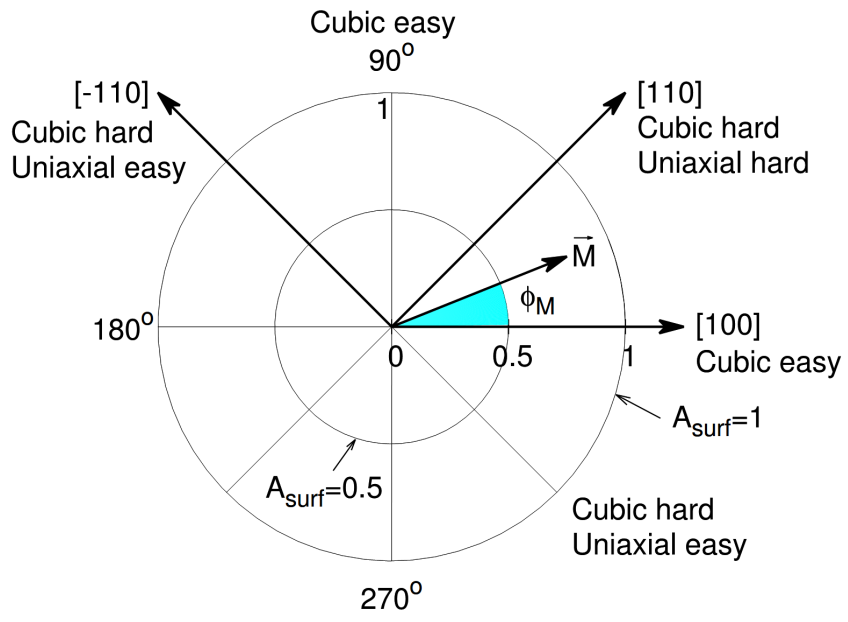

Fig. 1. The idea of surface pinning diagram: each point in the plane represents the surface pinning conditions described by a specific value of the surface pinning parameter $A_{\text {surf }}\left(\varphi_{M}\right)$ corresponding to the given magnetization direction $\varphi_{M}$. The circle $A_{\text {surf }}\left(\varphi_{M}\right)=1$ corresponds to natural pinning conditions.

In this coordinate system the azimuth angle $\varphi_{M}$ describes the orientation of the magnetization of the sample with respect to a reference axis, which is the [100] crystal axis; the distance between a point in the diagram and the pole of the coordinate system measures the value of the surface pinning parameter $A_{\text {surf }}=A_{\text {surf }}\left(\varphi_{M}\right)$ corresponding to a given azimuth angle. Against the pinning diagram in Fig. 1 we have added the crystal axes characterizing the magnetic anisotropy in the (Ga,Mn)As sample used in the SWR study [4] (in which the SWR measurements were performed in the (001) inplane configuration).

Introduced in papers $[8,9]$, the concept of surface pinning parameter $A$ was proposed to describe the degree of freedom of surface spins in their precession. By definition the surface pinning parameter value $A=1$ corresponds to the natural freedom of the surface spins resulting from breaking their bonds with neighbors eliminated from the system by surface cut. This particular value of $A$ divides the $\left(A_{\text {surf }}\left(\varphi_{M}\right), \varphi_{M}\right)$ plane into two different regions: within the circle of radius $A_{\text {surf }}\left(\varphi_{M}\right)=1$, where only bulk spin-wave modes exist, and region beyond this circle, in which SSWMs exist in the spectrum of allowed modes (see also [10-12]).

The pinning diagram in Fig. 2 presents the configuration dependence of the surface parameter resulting from our formula (1) against the experimental data obtained by Liu et al. [4]; squares indicate the experimental surface parameter values corresponding to different configurations. The theory proves to fit very well the experiment. Moreover, as we will see below in Fig. 3, the theory reproduces faithfully the experimentally observed evolution of the SWR spectrum with the azimuth angle $\varphi_{M}$.

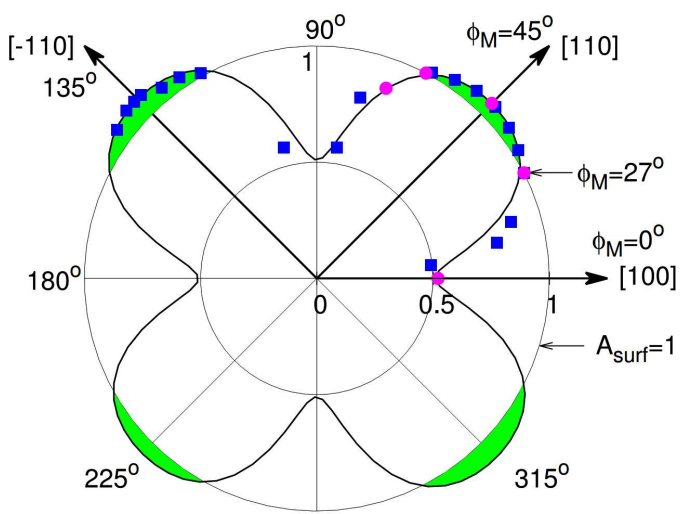

Fig. 2. Pinning trace, or the configuration dependence of the surface pinning, of a (Ga,Mn)As thin film plotted in the pinning diagram. The solid line represents the magnetization angle dependence of the in-plane surface pinning parameter resulting from our model (see Eqs. (1),(2)). This theoretical curve is found to fit very well the experimental data obtained by Liu et al. [4]; see the experimental points represented by squares.

Figure 3 shows spin-wave mode profiles resulting from our theory and the corresponding SWR spectra for five representative in-plane configurations that correspond to azimuth angles $\varphi_{M}=0^{\circ}, 27^{\circ}, 45^{\circ}, 62^{\circ}$ and $70^{\circ}$ (these configurations are also indicated in Fig. 2, where they are marked with red circles). The most important 
(a) $\phi_{\mathrm{M}}=0^{\circ} ; \mathrm{A}_{\text {surf }}=0.5213$
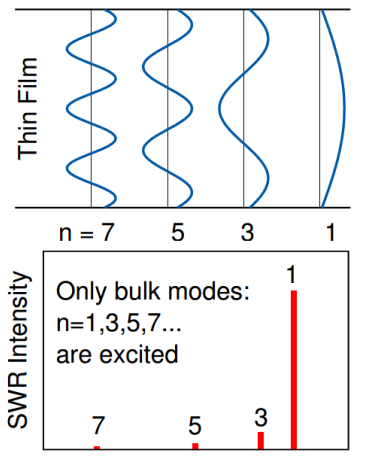

<--- Mode energy (arb. units)

(c) $\phi_{\mathrm{M}}=45^{\circ} ; \mathrm{A}_{\text {surf }}=1.0644$
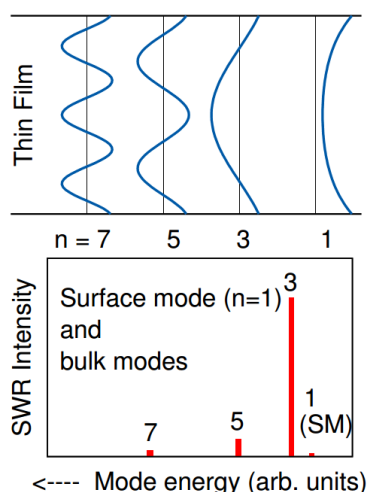

(b) $\phi_{\mathrm{M}}=27^{\circ}$ and $62^{\circ} ; \mathrm{A}_{\text {surf }}=1$
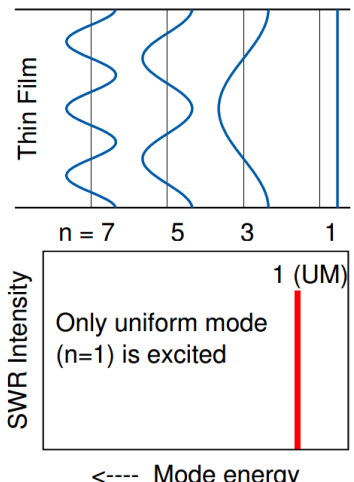

(d) $\phi_{\mathrm{M}}=70^{\circ} ; \mathrm{A}_{\text {surf }}=0.8700$
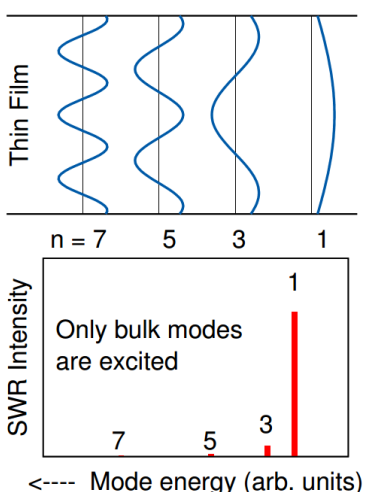

Fig. 3. Profiles of the lowest spin-wave resonance modes (top) and the corresponding SWR spectra (bottom) depicted separately for five in-plane configurations indicated by red points on the pinning trace in Fig. 2 . The spectra only exhibit peaks corresponding to symmetric modes of odd number, $n=1,3,5,7$. Two very peculiar effects are observed: firstly, the multi-peak SWR spectrum reduces to a single-peak FMR spectrum at two critical azimuth angles, $\varphi_{\mathrm{c} 1}=27^{\circ}$ and $\varphi_{\mathrm{c} 1}=62^{\circ}$; secondly, in the angle range bounded by these two critical angles the spectrum includes a surface-localized resonance peak.

feature of the configuration evolution that the experimental SWR spectrum undergoes as the azimuth angle increases to reach the consecutive values mentioned above is that from a multi-peak SWR spectrum at $\varphi_{M}=0^{\circ}$ (with $A_{\text {surf }}<1$ ) consisting exclusively of bulk modes, it becomes a single-peak FMR spectrum at the critical angle $\varphi_{\mathrm{c} 1}=27^{\circ}$ (corresponding to $A_{\text {surf }}=1$ ), and then, as the azimuth angle continues to grow above this critical value, regains its previous multi-peak form in the azimuth angle range $27^{\circ}<\varphi_{M}<62^{\circ}$, in which, however, it now includes a resonance peak corresponding to a surface mode (see the case with $\varphi_{M}=45^{\circ}$; $\left.A_{\text {surf }}\left(\varphi_{M}=45^{\circ}\right)>1\right)$. This result is a strong evidence that the SWR spectra measured in the in-plane configuration by Liu et al. [4] fulfill perfectly the assumptions of the surface inhomogeneity model, in which a key role is played by the surface magnetic anisotropy, constituting the source of the above-discussed effects.

\section{Factors responsible for surface pinning and surface mode existence}

Figure 4a shows two curves, each representing the azimuth angle dependence of a part of surface pinning resulting from selected anisotropies - the total contribution of the isotropic and uniaxial anisotropies is analyzed independently of the pinning related to the cubic anisotropy. The first two contributions consonantly

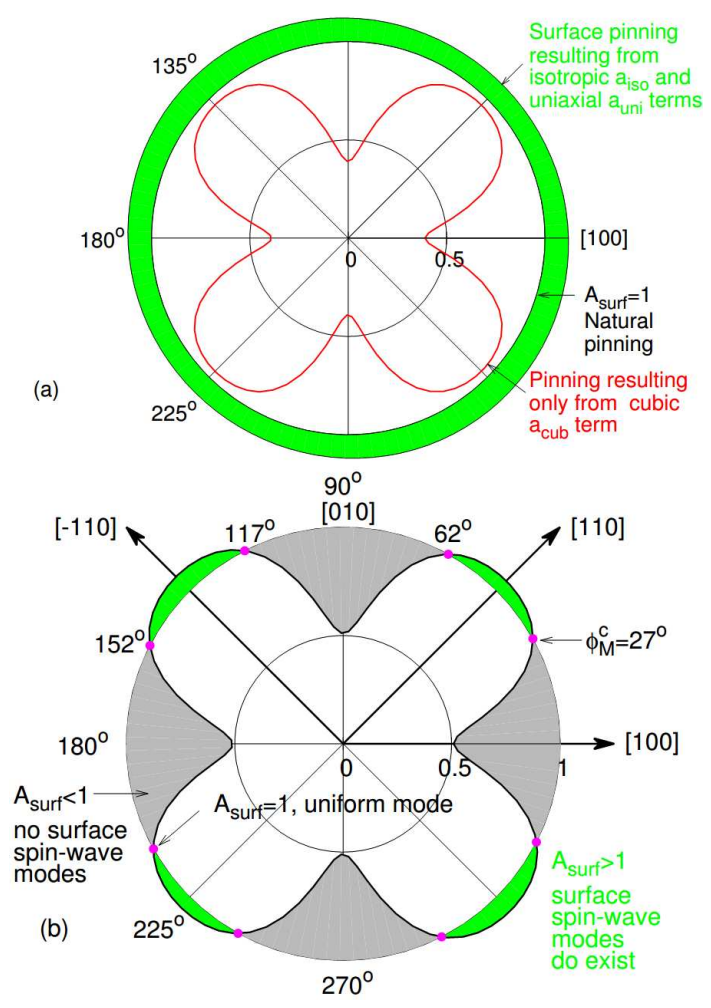

Fig. 4. Graphical analysis of the configuration evolution of the in-plane surface spin pinning in a $(\mathrm{Ga}, \mathrm{Mn}) \mathrm{As}$ thin film as described by Eqs. (1),(2). (a) Configuration dependence of two surface spin pinning contributions resulting from different factors considered separately (as indicated in the graph). (b) Represented by the solid line, the full surface pinning parameter $A_{\text {surf }}\left(\varphi_{M}\right)$ as a function of the in-plane azimuth magnetization angle $\varphi_{M}$; regions of existence of SSWMs under these full pinning conditions are marked in green.

introduce the pinning to that region of the diagram in which SSWMs exist for any azimuth orientation (green ring in the diagram). In contrast, the pinning resulting from the cubic anisotropy, represented by a quadrifoliumlike curve, is completely embedded in the region within the circle of radius $A_{\text {surf }}\left(\varphi_{M}\right)=1$; however, since the circle $A_{\text {surf }}\left(\varphi_{M}\right)=1$ corresponds to the natural pinning, this implies that the cubic anisotropy alone will not induce SSWMs in any azimuth orientation. Thus, already at this point, before proceeding to the analysis of the full pinning diagram presented in Fig. 4b, we must realize that it will result from two above-mentioned opposite tendencies. 
As a result of the summation of the above-mentioned pinning contributions with different effects on the spins - one increasing their freedom, the other reducing it in the full pinning diagram (Fig. 4b) the green ring is reduced to four islands that still remain within the region of surface mode existence. Each of these islands is associated with one hard cubic anisotropy axis, as indicated in Fig. 1. Also, each island corresponds to an azimuth angle range between the two critical angles for which $A_{\text {surf }}\left(\varphi_{M}\right)=1$; each of these angle ranges surrounds symmetrically the cubic anisotropy axis. It is in these azimuth angle ranges that SSWMs exist. Beyond them the cubic anisotropy has a destructive effect on the surface modes; this destruction is apparent in regions symmetric with respect to the easy cubic anisotropy axes.

Now we can provide physical grounds to these observations. A hard magnetic axis is a direction in which spins are reluctant to align, and setting them in an equilibrium direction involves a high energy cost to the system. Thus, the opposite tendency - divergence from the hard axis - must be more favorable energetically. This implies that a hard cubic anisotropy axis plays the role of an easy axis for the spin pinning; we can refer to it as an easy pinning axis, i.e., an axis from which spins diverge easily, since their pinning along its direction is weaker. And vice versa: an easy cubic anisotropy axis defines a direction that spins are reluctant to quit - a hard surface pinning axis that hampers the occurrence of SSWMs. Thus, the following final conclusion can be drawn from our considerations based on the pinning diagram: in a (Ga,Mn) As thin film conditions favorable for the occurrence of surface spin-wave modes in the in-plane configuration are fulfilled first of all for those azimuth orientations of the magnetization of the sample that lie around the hard axes of cubic magnetic anisotropy.

\section{Acknowledgments}

This study is a part of a project financed by Narodowe Centrum Nauki (National Science Centre of Poland), grant no. DEC-2013/08/M/ST3/00967.

\section{References}

[1] J. Kanski, L. Ilver, K. Karlsson, M. Leandersson, I. Ulfat, J. Sadowski, Electronic structure of $(G a, M n)$ As revisited: an alternative view on the "Battle of the bands", arXiv:1410.8842v2 [cond-mat.mtr]sci].

[2] M. Dobrowolska, K. Tivakornsasithorn, X. Liu, J.K. Furdyna, M. Berciu, K.M. Yu, W. Walukiewicz, Nature Mater. 11, 444 (2012).

[3] N. Samarth, Nature Mater. 11, 360 (2012).

[4] X. Liu, Y.-Y. Zhou, J.K. Furdyna, Phys. Rev. B 75, 195220 (2007).

[5] H. Puszkarski, P. Tomczak, Sci. Rep. 4, 6135 (2014).

[6] H. Puszkarski, P. Tomczak, Phys. Rev. B 91, 195437 (2015).

[7] H. Puszkarski, P. Tomczak, Acta Phys. Pol. A 127, 508 (2015).

[8] H. Puszkarski, Acta Phys. Pol. A 38, 217 and 899 (1970).

[9] H. Puszkarski, Prog. Surf. Sci. 9, 191 (1979).

[10] L. Wojtczak, Magnetic Thin Films, Wydawnictwo Uniwersytetu Łódzkiego, Łódź 2009 (in Polish).

[11] A. Urbaniak-Kucharczyk, Phys. Status Solidi B $\mathbf{1 8 9}$, 239 (1995).

[12] Diep-The-Hung, J.C.S. Lévy, Surf. Sci. 80, 512 (1979). 